\title{
Les thrombopathies constitutionnelles
}

Qu'elles soient dues à une anomalie membranaire ou intracellulaire, les thrombopathies constitutionnelles constituent un excellent modèle d'étude de la physiologie plaquettaire.

Sylvia Bellucci

Assistant des hôpitaux et des Universités.

\section{Jacques Caen}

Professeur à la faculté Lariboisière-Saint-Louis-Université Paris-VII.

Chef $d u$ service d'immunohématologie à l'hôpital Lariboisière. Directeur de l'unité Inserm $U$ I5O et du laboratoire LA 334 du Cnrs.

\section{REFERENCES}

1. Bernard J, Soulier JP. Sur une nouvelle variété de dystrophie thrombocytaire hémorragique congénitale. Sem Hop Paris 1948; 24: 3217-23. 2. Caen J, Levy-Toledano S. Interaction between platelets and von Willebrand factor provides a new scheme for primary hacmostasis. Nature 1973; 244: 1 59-6o.

3. Fauvel F, Grant ME, Legrand YJ, et al. Interaction of blood platelets with a microfibrillar extract from adult bovine aorta: requirement for von Willebrand factor. Proc Natl Acad Sci USA $1983 ; 80: 551-4$.

4 Nurden AT, Caen JP. Specific roles for surface glycoproteins in platelet function. Nature 1975 ; 255: 720-2.

\section{ADRESSE}

S. Bellucci, J.P. Caen : département d'angiohématologie, hôpital Lariboisière, 6, rue Guy-

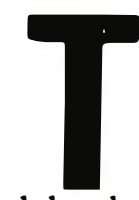

oute plaie de la paroi vasculaire entraîne un syndrome hémorragique qui est tari grâce à l'intervention de minuscules cellules du sang périphérique : les plaquettes, dépourvues de noyau et d'un diamètre de l'ordre de 3 microns. Et ceci, conjointement aux phénomènes de coagulation plasmatique. Que la fonction des plaquettes vienne à être perturbée, nous sommes alors en présence de manifestations hémorragiques, parfois particulièrement alarmantes. Certains stimuli provenant de la lésion de l'endothélium vasculaire (ADP - thrombine - exposition du collagène $\mathrm{du}$ subendothélium...) vont être responsables de l'entrée en scène de ces acteurs protagonistes de l'hémostase primaire que sont les plaquettes. Pour éviter toute complication hémorragique, les différentes étapes de la physiologie plaquettaire : adhésion au subendothélium, activation, sécrétion du contenu granulaire, agrégation nécessitent un déroulement complexe et harmonieux. C'est à l'échelle de la biologie moléculaire que celui-ci est maintenant connu. Et si cette compréhension, chaque jour plus fine, doit beaucoup aux travaux de recherche fondamentale, elle a été aussi largement tributaire de l'étude approfondie de certains désordres fonctionnels au premier rang desquels émergent les thrombopathies constitutionnelles. Plutôt que d'en constituer une revue exhaustive, cet article aura surtout pour but de préciser ce que l'étude de ces affections apporte à la connaissance de la physiologie de l'hémostase, afin d'en souligner l'extraordinaire richesse (tableau I et figure I).

\section{Trouble de l'adhésion des plaquettes}

I. Le syndrome de BernardSoulier ou dystrophie thrombocytaire hémorragipare. Décrite en 1948, par J. Bernard et J.P. Soulier [1], cette thrombopathie est caractérisée par un allongement du temps de saignement, une thrombopénie modérée, l'existence de plaquettes géantes sur lame, une consommation de la prothrombine défectueuse alors que la rétraction du caillot est normale. La transmission génétique est autosomale, incomplètement récessive. Les manifestations hémorragiques cutanéomuqueuses sont constantes, d'intensité variable mais souvent sévères, responsables d'un taux de mortalité estimé aux alentours de seize pour cent.

L'hémogramme met en évidence une thrombopénie initialement modérée mais qui s'aggrave au cours du temps et dont le mécanisme reste encore incompris. Les plaquettes ont une taille augmentée, pouvant atteindre un diamètre de $30 \mu$ et leurs granulations sont rassemblées au centre de la cellule offrant un aspect pseudolymphocytaire. Les études en microscopie électronique montrent un système membranaire très anormal : tor- 
tueux, très développé. Les mêmes anomalies portant également sur les membranes de démarcation sont retrouvées au niveau des mégacaryocytes. Les tests fonctionnels plaquettaires montrent une agrégation normale en présence d'ADP, de collagène, d'adrénaline ou d'acide arachidonique; en présence de thrombine l'agrégation est retardée, encore plus en présence de ristocétine; enfin, en présence de facteur VIII/von Willebrand bovin, l'agrégation est nulle [2]. L'adhésion $\mathrm{au}$ collagène est normale mais l'adhésion au subendothélium, jugée par la technique de Baumgartner sur aorte de rat désendothélialisée, est très diminuée. Or, le facteur VIII/von Willebrand est nécessaire à une telle adhésion, l'élément du subendothélium mis en jeu semblant être les microfibrilles [3]. L'hypothèse selon laquelle les plaquettes du syndrome de BernardSoulier sont dépourvues du récepteur nécessaire à la fixation du facteur VIII/von Willebrand plasmatique fut dès lors envisagée. Le déficit moléculaire membranaire présenté par ces plaquettes était simultanément précisé. La glycoprotéine $\mathrm{Ib}$, par électrophorèse sur gel SDS de polyacrylamide est indétectable au niveau de la membrane [4]. D'autres déficits en glycoprotéines V, IX, 100, probablement liés à la glycoprotéine Ib ont été décrits depuis. Les études immunologiques utilisant des anticorps polyclonaux ou monoclonaux dirigés contre la glycoprotéine Ib ont été riches en renseignements. Par immunoélectrophorèse en double dimension, le défaut en glycoprotéine Ib a pu être confirmé. Surtout, il a pu être montré que la glycoprotéine Ib était le récepteur principal du facteur VIII/von Willebrand plasmatique [5]; de telles études ont également mis en évidence l'implication de la glycoprotéine $\mathrm{Ib}$, avec la glycoprotéine $\mathrm{V}$, dans la fixation de la thrombine à la plaquette, expliquant ainsi le retard d'agrégation en présence de thrombine et, peut-être, le défaut de consommation de la prothrombine noté dès la description princeps.

Par immunofluorescence ou radioimmunoassay, la fixation de tels anticorps peut être également
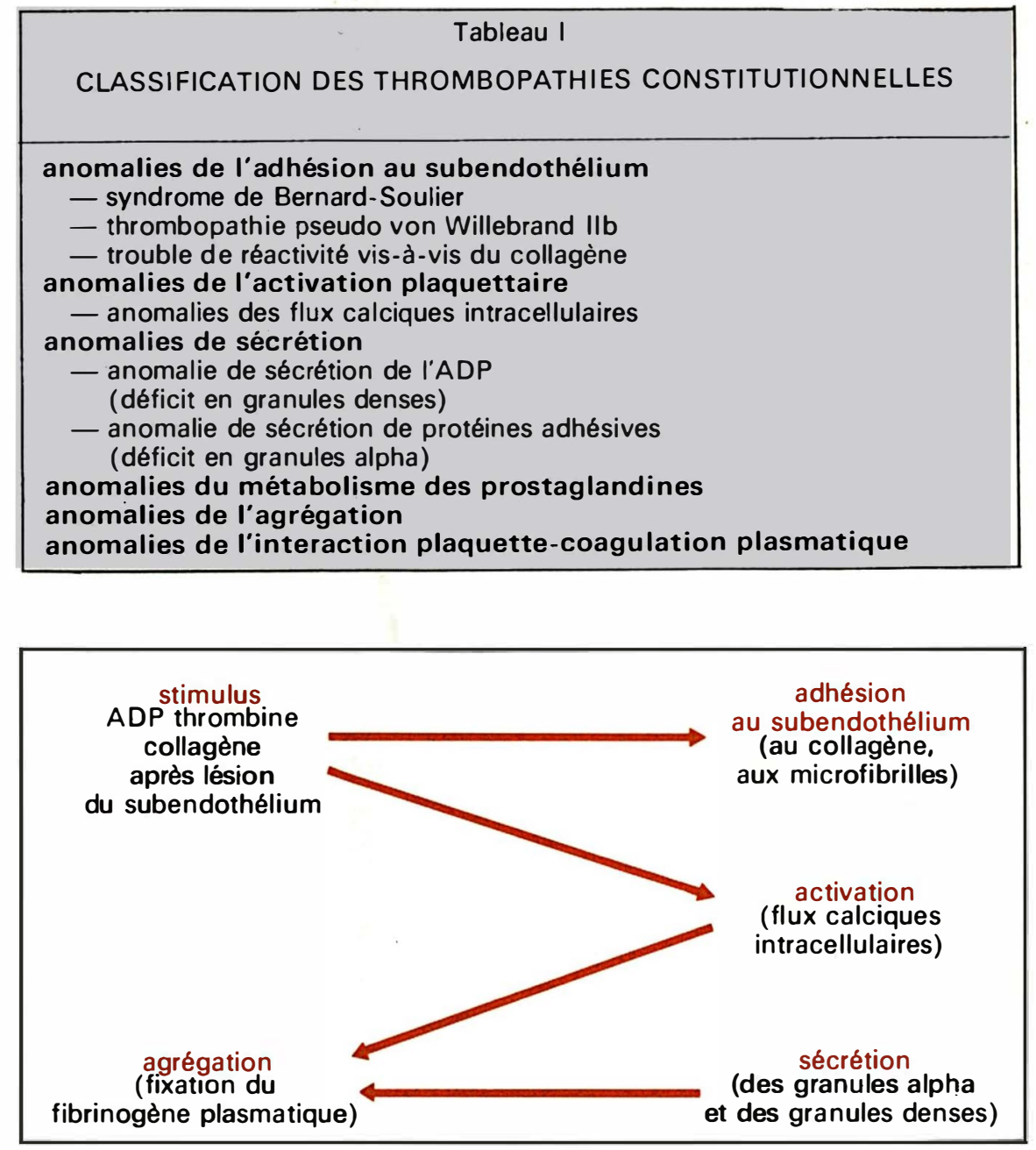

Figure I. Schéma des différentes élapes de la physiologie plaquellaire.

étudiée; ces tests permettent le dépistage des sujets hétérozygotes et le diagnostic anténatal de l'affection. Celui-ci devrait être encore facilité par le clonage du gène codant pour la glycoprotéine Ib dans un avenir proche.

Ainsi, au cours du syndrome de Bernard-Soulier, pour la première fois un défaut structural membranaire, le déficit en glycoprotéine $\mathrm{Ib}$, explique une anomalie fonctionnelle : le défaut d'adhésion des plaquettes au subendothélium.

2. La thrombopathie pseudo von Willebrand IIb. Ici encorc, la thrombopathic est accompagnéc d'une thrombopénie volontiers oscillante. Les plaquettes sur lame ont tendance à s'agglutiner et ont une taille augmentéc. Le taux du factcur VIII coagulant, du facteur VIII/von Willebrand (mesuré immunologiquement ou par son activité cofactcur de la ristocétinc) cst diminué au nivcau plasmatique : cette diminution est duc à une captation accrue au niveau de la membrane plaquettaire [6], du fait d'une concentration augmentée de glycoprotéinc Ib [7]. Ce tableau est donc exactement l'inverse de celui réalisé au couı du syndrome de Bernard-Soulicr. Les plaquettes possèdent ainsi une quantité accrue de factcur VIII/von Willebrand à lcur surface; clles ont tendance à s'agglutiner ct à avoir une durée de vic diminuéc, ce fait pouvant expliquer la thrombopénic obscrvéc. Une qualité moindre et une quan- 
tité moins grande de facteur VIII/ von Willebrand plasmatique sont disponibles pour se lier normalement à la fois aux plaquettes et au subendothélium en cas de lésion de l'endothélium. Les manifestations hémorragiques, l'allongement du temps de saignement sont ainsi probablement dus à un défaut d'adhésion de ces plaquettes fragilisées au subendothélium. Un tel défaut reste toutefois à démontrer précisément au cours de ce syndrome.

A l'heure actuelle, ce syndrome pseudo-von Willebrand IIb nous permet de souligner l'importance d'une relation quantitativement et qualitativement normale entrc la plaquette d'une part et le facteur VIII/von Willebrand plasmatique d'autre part, dans le phénomène d'adhésion.

3. Thrombopathies par défaut de réactivité vis-à-vis du collagène. Parfois, lors de l'étude fonctionnelle des plaquettes d'un patient présentant des manifestations hémorragiques, la seule anomalie retrouvée est un défaut d'agrégation vis-à-vis du collagène. Une étude plus fine de ces cas serait nécessaire, portant en particulier sur l'étape d'adhésion vis-à-vis du collagène. Là encore, une bonne description du ou des défauts moléculaires responsables de ces thrombopathies permettra de micux comprendre une étape clé de la physiologie plaquettaire. Une anomalie du récepteur du collagène, peut-être en relation avec un déficit en glycoprotéine Ia, a par exemple été évoquée, clle a même été démontrée récemment chez un patient [8].

Ainsi, ces thrombopathies sont-elles particulières en ce qu'elles permettent de mieux analyser la relation plaquette-collagène, relation qui permet, semble-t-il, une adhésion au subendothélium sans l'intervention du facteur VIII/von Willebrand.

L'adhésion au subendothélium constitue donc une étape fonctionnelle mettant en jeu, dans les conditions normales, surtout les plaquettes et, à un degré moindre, certaines cellules sanguines; son exagération peut être à l'origine des maladies thrombotiques. Au cours d'autres conditions pathologiques, l'adhé-

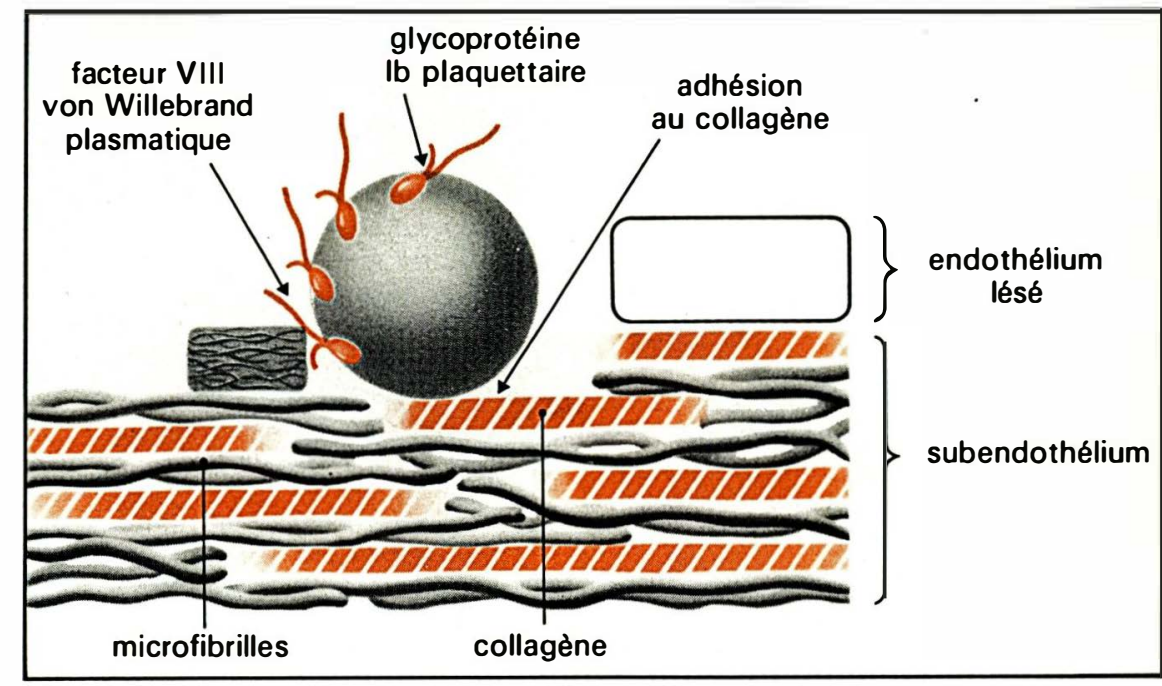

Figure 2 a. Adhésion plaquetlaire normale.

sion peut intéresser d'autres cellules comme les cellules tumorales qui favorisent le développement de métastases. Inversement, le défaut d'adhésion peut certes intéresser la relation plaquette-paroi vasculaire à l'origine de manifestations hémorragiques, mais aussi la relation cellule-cellule venant détruire la structure tissulaire, comme cela est observé très tôt au cours de la transformation maligne. C'est en ce sens qu'une meilleure connaissance de ce phénomène peut permettre des progrès, non seulement en hémostase mais encore en cancérologie (figure $2 a$ et $2 b$ ).

\section{Défaut}

d'activation plaquettaire

Certaines thrombopathies sont caractérisées par un défaut d'agrégation vis-à-vis des différents agents inducteurs alors que les structures membranaires et granulaires semblent préservées. La mesure possible des constituants intragranulaires (bétathromboglobuline présente dans les granules alpha, ADP présent dans les granules denses), montre un taux normal de ceux-ci éliminant de façon certaine une thrombopathie par déficit granulaire de type " pool vide ». Par ailleurs, après stimulation par l'acide arachidonique, on observe une synthèse de thromboxane $\mathrm{A} 2$, prostaglandine principale d'origine plaquettaire, témoignant de la normalité de cette voie métabolique.
Dès lors s'impose l'étude en présence de ionophore A 23187, qui permet normalement le flux de calcium $\mathrm{Ca}^{++}$des sites de stockage vers le cytoplasme pour entraîner activation et agrégation [9]. Au cours de ces thrombopathies, l'agrégation est nulle en présence de ionophore calcique A 23187 , orientant vers une anomalie de flux calciques intracellulaires nécessaires à l'activation. Une telle hypothèse peut être vérifiée par le test utilisant le QUIN 2 dont l'estérification consécutive aux flux calciques intracellulaires se traduit par un changement de fluorescence, absent au cours de ces affections. Normalement, l'augmentation du calcium intracytoplasmique, à partir des sites de stockage (vésicules du système tubulaire dense, membrane plasmique) aboutit à plusieurs événements cellulaires, regroupés sous le terme d'activation plaquettaire [10]. Ainsi se produit l'activation de la chaîne légère de la myosine, entraînant la phosphorylation d'une protéine de poids moléculaire 20000 (P 20000), ainsi se produit également la phosphorylation d'une protéine de poids moléculaire 47000 (P 47000$)$, grâce à l'activation de la protéine kinase $\mathrm{C}$. Ces protéines phosphorylées $\mathbf{P} 20000$ et $\mathrm{P} 47000$ sont responsables respectivement de la fusion des granules et de la sécrétion de leurs constituants [11]. L'activation de la myosine permet d'autre part le changement de forme et la contraction. L'on 


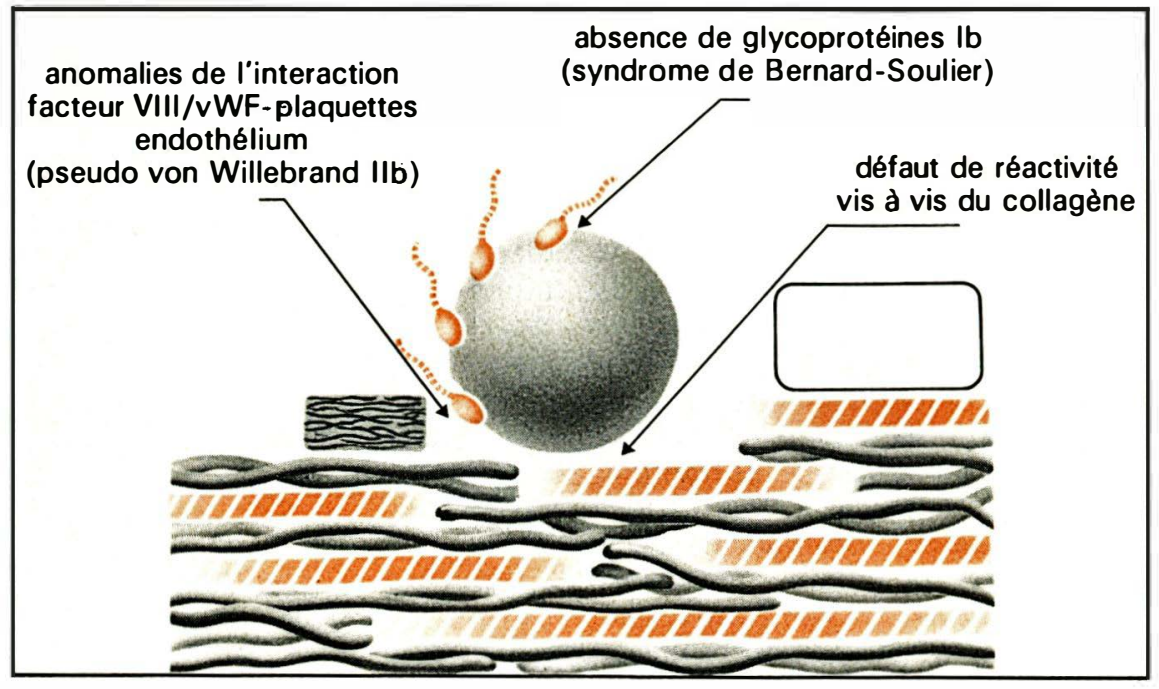

Figure $2 \mathrm{~h}$. Thrombopalhies par adhésion plaquellaire anormale. Facteur $V I I I / v W F=V I I I /$ von Willebrand.

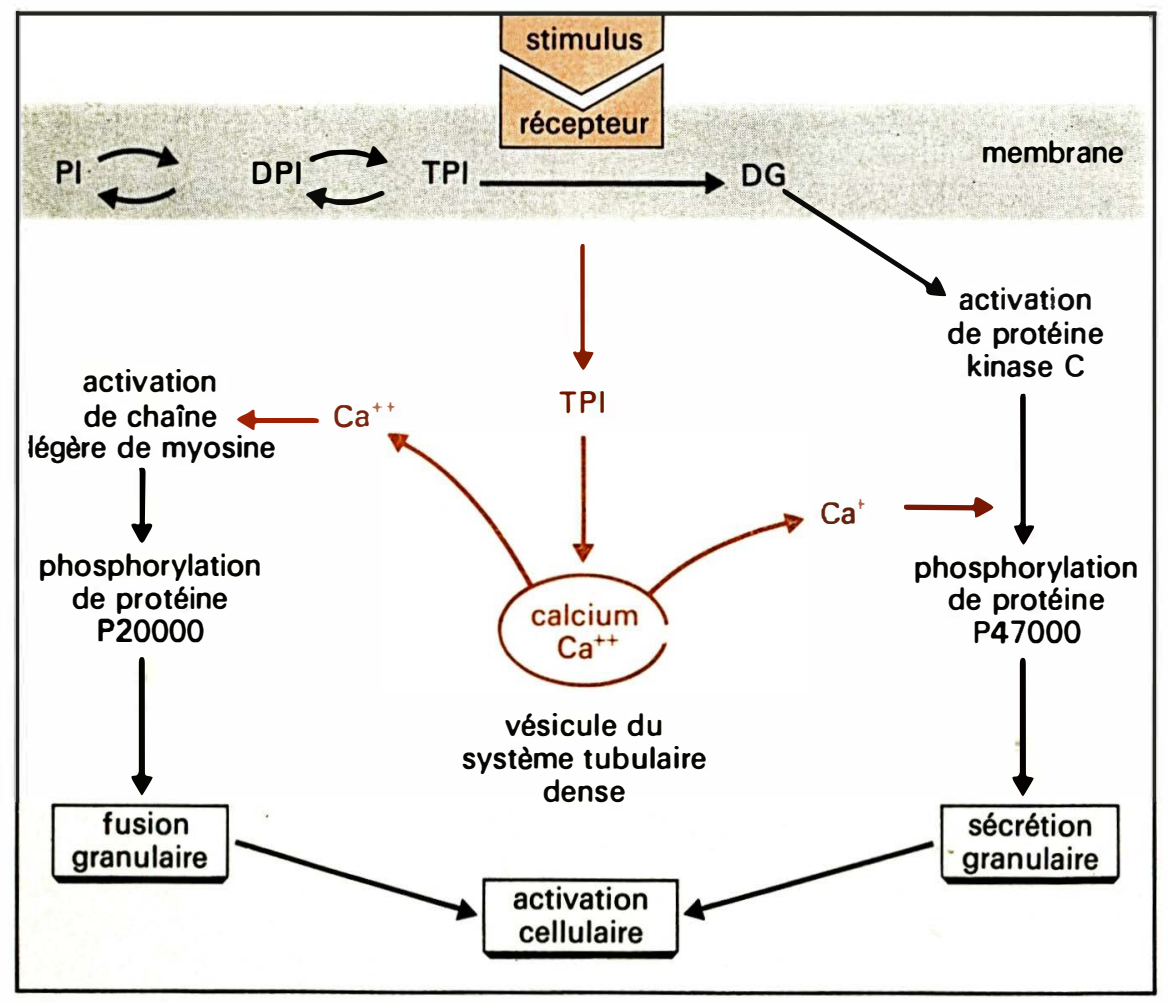

Figure 3. Rôle des flux calciques el du mélabolisme des triphosphoinositide's dans l'actization cellulaire.

$P I=$ phosphoinositol $; D P I=$ diphosphoinositol; $T P I=$ triphosphoinosilol.

$m / s n^{\circ} 8$ decembre 85 comprend dès lors qu'en cas d'anomalies des flux calciques intracellulaires, ces thrombopathies se manifestent par un changement de forme défectueux, une absence de sécrétion granulaire et finalement un défaut d'agrégation. De telles thrombopathies viennent d'être décrites récemment $[12,13]$. Leur recherche plus systématique et leur reconnaissance plus fréquente devraient permettre d'analyser précisément chaque étape du rôle du calcium intracellulaire. Le fait que certaines de ces thrombopathies aient été rapportées chez des patients présentant par ailleurs des troubles du comportement [13] n'est pas sans rappeler la similitude structurale et fonctionnelle qui existe entre la plaquette et la cellule nerveuse.

L'activation plaquettaire met également en jeu des modifications de structure des phospholipides intramembranaires, marquées essentiellement par une chute brutale des triphosphoinositides (TPI) [14]. De telles anomalies ont été décrites conjointement à l'existence d'anomalies, granulaires par exemple. Pourraient-elles réaliser à elles seules une thrombopathie authentique ? Cette hypothèse reste encore à démontrer.

L'étude plus approfondie de ce type de thrombopathies marquées par des anomalies des flux calciques et/ou du métabolisme des phospholipides membranaires, devrait nous permettre de mieux comprendre le phénomène crucial d'activation cellulaire au niveau de la plaquette, mais aussi d'autres cellules, présentant une similitude certaine comme la cellule nerveuse (figure 3).

\section{Défaut \\ de sécrétion d'ADP}

Certaines thrombopathies sont marquées par une diminution de l'agrégation en présence d'ADP et d'adrénaline qui ne provoquent pas, par ailleurs, de deuxième vague d'agrégation. En réponse au collagène ou à l'acide arachidonique, l'agrégation est également diminuée [15]. Ces anomalies peuvent être en rapport avec une réduction du nombre de granules denses de ces plaquettes, estimé par microscopic 


\section{REFERENCES}

5. Ruan C, Tobelem G, McMichael AJ, et al. Monoclonal antibody to human platelet glycoprotein I. Effects on human platelet function. $\mathrm{Br} \mathcal{J}$ Haematol 1981; 49: 511-9.

6. Weiss $\mathrm{HJ}$, Meyer D, Rabinowitz $\mathrm{R}$, et al. Pseudo von Willebrand's disease. An intrinsic platelet defect with aggregation by unmodified human factor VIII/von Willebrand factor and enhanced adsorption of its high molecular weigh multimers. $N$ Engl 7 Med 1982 ; 306: 326-33

7. Bryckaert MC, Pietu G, Ruan C, et al. Abnormality of glycoprotein $\mathrm{Ib}$ in two cases of pseudo von Willebrand disease. $7 \mathrm{Lab}$ Clin Med 1985 (sous presse)

8. Nieuwenhuis HK, Akkerman JWN, Sakariassen KS, Houdijk WPM, Nievelstein PFEM

Sixma JJ. Human blood platelets with a def ect of response to collagen fail to express surface glycoprotein Ia. Thrombosis and Haemostasis 1985; 54 733 A (Abstract).

9. Levy-Toledano S, Maclouf J, Rendu F, Rigaud M, Caen JP. Ionophore A 23187 and thrombasthenic platelets. Dissociating serotonin release and thromboxane formation from truc aggregation. Thromb res 1979; 16: 453-62.

10. Levy-Toledano S, Maclouf J, Bryon P, Savariau E, Hardisty RM, Caen JP. Human platelet activation in the absence of aggregation: a calcium dependent phenomenon independent of thromboxane formation. Blood $1982 ; 59$ : 1078-85

11. Gerrard JM, Isracls SJ, Friesen LL. Protein phosphorylation and platelet secretion. Nouv Rev Fr Hematol/Blood Cells, 1985 (sous presse)

12. Hardisty RM, Machin SJ, Nokes TJC, Pink $T J$, Sharon W. A new congenital def ect of platele secretion: impaired responsiveness of the platelets to cytoplasmic free calcium. $\mathrm{Br} J \mathrm{Haematol} 1983$; 53: 543-57.

13. Koike K, Rao AK, Holmsen H, Mueller PS. Platelet secretion defect in patient with the attention deficit disorder and easy bruising. Blood $1983 ; 63$ : 427-33.

14. Rendu F, March P, Maclouf J, Girard A, Levy-Toledano S. Triphosphoionositide breakdown and dense body release as the earliest events in thrombin induced activation of human platelets. Biochem Biophys Res Commun 1983; 116 513-9.

15. Hardisty RM, Hutton RA. Bleeding tendency associated with "new " abnormality of platele behaviour. Lancet 1967; i: 983-5.

16. Weiss $\mathrm{HJ}$. Congenital disorders of platelet function. Semin Hematol 1980; 17: 228-41.

17. Born GVR. Aggregation of blood platelet by adenosine diphosphate and its reversal. Nature 1962 ; 194: 927-31.

18. Malmsten C, Hamberg $M$, Svenson J Samuelsson B. Physiological role of an endoperoxide in human platelets: Hemostatic def ect due to platelet cyclo-oxygenase deficiency. Proc Natl Acad Sci USA 1975; 72: 1446-50.

19. Defreyn G, Machin SJ, Carreras LO, Dauden MV, Chamone DAF, Vermylen J. Familia bleeding tendency with partial platelet thromboxane synthetase deficiency: reorientation of cyclic endoperoxide metabolism. Br 7 Haematol 1981 ; 49: 29-41.

20. Raccuglia G. Gray platelet syndrome. A variety of qualitative platelet disorder. $A m \mathcal{J} \mathrm{Med}$ $1971 ; 51$ : 818-28. électronique ou par le test de mépacrine fluorescente : elles réalisent les thrombopathies de type pool vide [I6]. Les granules denses contiennent l'ADP qui constitue la voie métabolique principale de l'agrégation [17]; physiologiquement cette voie est mise en jeu après lésion de l'endothélium, lorsque les plaquettes sont au contact de l'ADP issu des cellules endothéliales lésées, du collagène présent dans le subendothélium ou de la thrombine formée au cours de la coagulation. Au cours de ces thrombopathies, il existe donc une diminution de la sécrétion d'ADP, consécutive au déficit en granules denses, et mesurable directement après action des agents agrégants. De telles thrombopathies sont exceptionnelles et parfois associées à un déficit en granules alpha, que nous décrirons plus loin. Elles peuvent constituer enfin, un des éléments d'autres syndromes constitutionnels, marqués en outre par un déficit immunitaire (syndrome de Chediak-Higashi, syndrome de Wiskott Aldrich...) sans qu'il soit encore possible d'établir un lien entre ces manifestations.

Les nouvelles techniques d'investigation ont actuellement pour objet d'étudier, au cours de ces thrombopathies, la libération des constituants des granules alpha, la synthèse de prostaglandines, les phénomènes d'activation (mobilisation du calcium intracellulaire, phosphorylation de protéines, modifications des phospholipides membranaires), afin d'estimer le rôle des constituants des granules denses (et en premier lieu celui de l'ADP) au cours de ces événements.

Ces thrombopathies, marquées par une réduction du nombre des granules denses, apparaissent comme un modèle d'étude privilégié pour la reconnaissance de certaines régulations : celles-ci peuvent intéresser, au sein d'une même étape fonctionnelle, (comme l'agrégation) l'intrication, par exemple des voies métaboliques concernées; par la mise en jeu de réactions biochimiques complexes, ces régulations peuvent aussi intéresser plusicurs étapes : activation, sécrétion, étapes clés de la physiologic plaquettaire (voir figures $4 a$ et $4 b$ ci-contre).

A l'état normal, après action d'un stimulus comme la thrombine ou le collagène, les phospholipides membranaires sont hydrolysés par des phospholipases et libèrent l'acide arachidonique. Grâce à une enzyme, la cyclooxygénase, celui-ci est transformé en endoperoxyde, puis, grâce à la thromboxane synthétase, en thromboxane $A_{2}$, prostaglandine principale des plaquettes.

\section{Troubles de synthèse des prostaglandines}

Par ailleurs, des isomérases peuvent transformer l'endoperoxyde en d'autres prostaglandines : $\mathrm{PGF}_{2} \alpha$, $\mathrm{PGD}_{2}$ antiagrégante, $\mathrm{PGE}_{2}$ proagrégante. D'exceptionnels déficits portant, soit sur la cyclooxygénase [18], soit sur la thromboxane synthétase [19] ont été rapportés, où les plaquettes présentent un défaut d'agrégation en présence de collagène, de thrombine et d'acide arachidonique. L'étude de la réponse en présence d'endopéroxyde et celle de l'équilibre du taux des diverses prostaglandines formées, permettent de distinguer ces deux déficits entre eux.

De telles thrombopathies réalisent à l'état constitutionnel un tableau analogue à celui que l'on peut observer après prescription d'inhibiteurs de la cyclooxygénase (au premier rang desquels l'aspirine), ou plus récemment après administration d'antagonistes de la thromboxane synthétase : ces deux types de drogues constituent des armes majeures de l'arsenal thérapeutique actuel antithrombotique (figures $4 a$ et $4 b$ ).

\section{Syndrome des plaquettes grises}

La thrombopathic par déficit de sécrétion de protéines endogènes favorisant le contact cellulaire, ou syndrome des plaquettes grises, a été décrite dès I97I par Raccuglia [20]. Cette thrombopathic exceptionnelle associe à une thrombopénic modéré, une thrombopathic caractérisée par l'existence de plaquettes de taille légèrement augmentée et surtout dépourvues de granulations azurophiles, normalement visibles par la coloration de May-Grunwald Giemsa. Le terme 


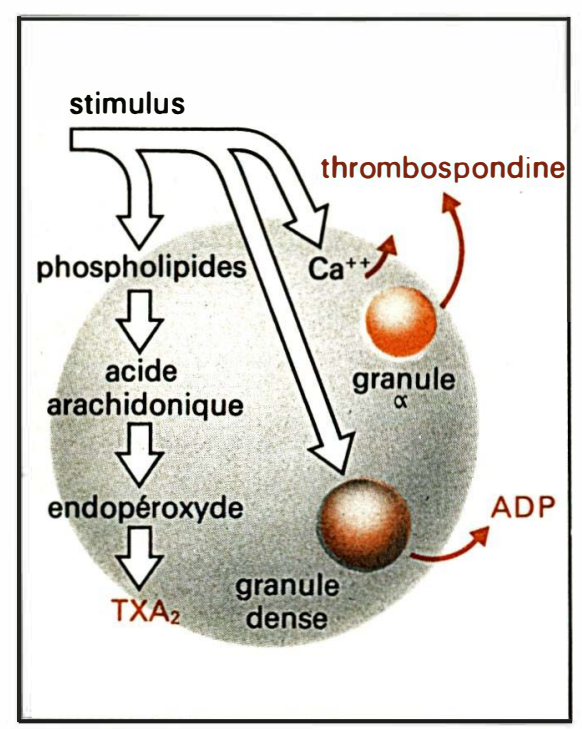

Figure 4a. Schéma sur le mélabolisme des prostaglandines el le mécanisme de sécrétion.

$T X A_{2}=$ thromboxane $A_{2}$.

de syndrome des plaquettes grises était donc retenu pour dénommer cette affection.

Les études ultrastructurales ont confirmé l'absence de granules alpha, tant au niveau des plaquettes, qu'au niveau des cellules précurseurs : les mégacaryocytes [21]. Les études fonctionnelles montrent, conjointement à une sécrétion diminuée du contenu des granules denses, une hypoagrégation, surtout en présence de collagène, d'adrénaline ou de thrombine [22]. Le taux intraplaquettaire des protéines normalement présentes dans les granules alpha est très diminué, voisin de $15 \%$ : c'est le cas du facteur VIII/ von Willebrand, du fibrinogène, de la fibronectine, du facteur 4 plaquettaire, de la bétathromboglobuline, ou du facteur mitogénique plaquettaire (PDGF) [23]. La structure glycoprotéinique membranaire est par contre normale.

La sécrétion de facteurs normalement contenus dans les granules alpha peut être importante : pour favoriser la formation de thrombine et la coagulation (rôle du facteur V, $\mathrm{du}$ fibrinogène intraplaquettaire), pour favoriser le contact cellulaire (rôle de la fibronectine, du facteur 4 plaquettaire) au sein de l'agrégat plaquettaire dès le stade le plus pré-

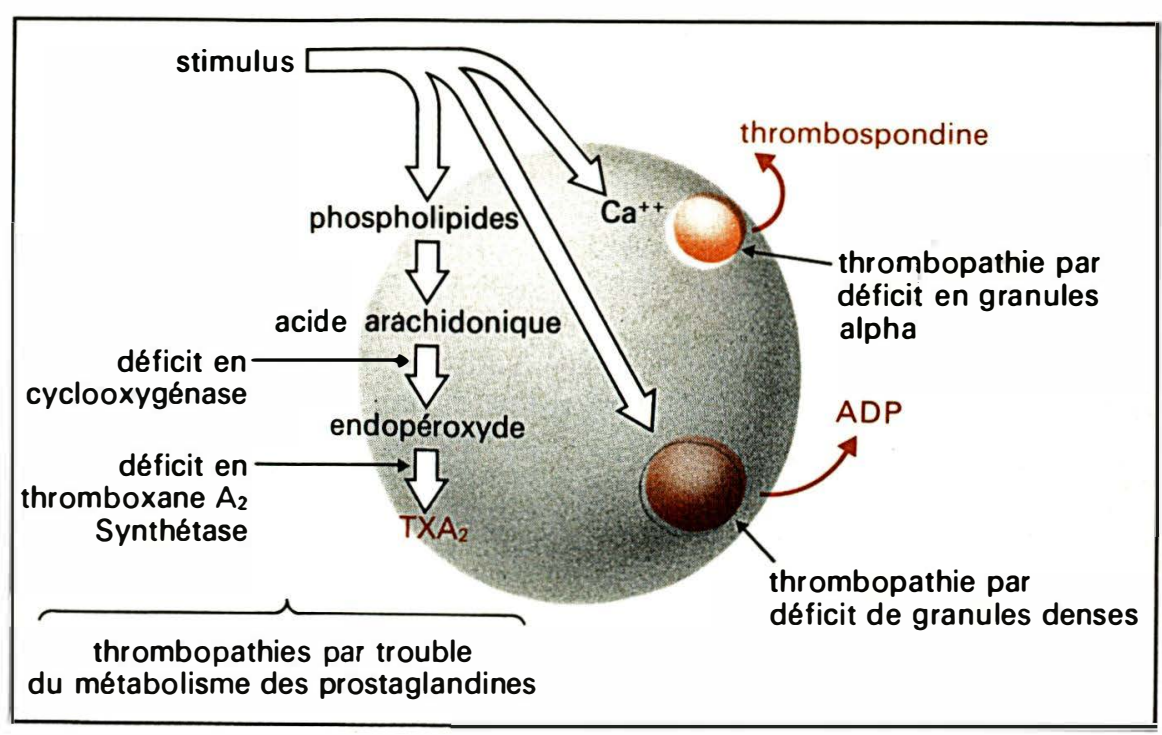

Figure ${ }_{4} b$. Thrombopalhies par anomalies du mélabolisme des prosıaglandines ou défaul de sécrétion.

coce de sa formation. Par ailleurs, la sécrétion du facteur VIII/von Willebrand ou de la fibronectine d'origine plaquettaire peut favoriser l'adhésion au subendothélium avant même la participation de ces mêmes facteurs d'origine plasmatique. La thrombospondine enfin, vient, après le stade de sécrétion, s'exposer à la membrane plaquettaire pour faciliter la fixation du fibrinogène plasmatique et favoriser ainsi l'agrégation [24]. Le syndrome hémorragique présenté par ces patients pourrait donc être en relation avec le défaut de libération de ces facteurs dont on commence à voir le rôle, lors de la formation de l'agrégat. Autre point intéressant à souligner au cours de cette thrombopathic: l'existence d'une myélofibrose siégeant électivement autour des mégacaryocytes [21]. Dans la genèse de celle-ci, on relève la forte probabilité d'une augmentation de certains facteurs au niveau des sinus médullaires, par défaut de stockage dans les granules alpha; citons ainsi le rôle d'une augmentation du facteur 4 plaquettaire à activité anticollagénase [25], ou surtout, l'intervention du facteur mitogénique plaquettaire dit PDGF qui favorise la prolifération de plusieurs types cellulaires dont les fibroblastes [26].
Plusicurs pôles d'intérêts s'offrent à l'étude plus fine de cette thrombopathic : quelle est l'importance de l'intervention des facteurs contenus dans les granules alpha au cours des différentes étapes de l'hémostase primaire? Existe-t-il une synergie fonctionnelle entre la libération de ces facteurs et celle d'autres composés présents, en particulier, au niveau des granules denses? En dehors de certaines circonstances pathologiques comme la myćlofibrose, quel peut être le rôle des facteurs de croissance comme le PDGF, stockés dans une cellule qui a perdu toute capacité de division? (figures $4 a$ et $4 b$ ).

\section{Thrombasthénies de Glanzmann}

Il s'agit en fait de la première thrombopathic par défaut d'agrégation décrite dès 1918 par le pédiatre suisse Glanzmann [27]. Celui-ci avait en effet été frappé par l'existence d'un désordre plaquettaire, marqué par un défaut d'agglutination sur lame et un défaut de rétraction du caillot, alors que la numération des plaquettes était normale. La transmission était autosomique récessive et le syndrome hémorragique cutanéomuqueux volontiers 


\section{REFERENCES}

21. Breton-Gorius J, Vainchenker W, Nurden A, Levy-Toledano S, Caen JP. Def ective $\alpha$-granule production in megakaryocytes from gray platelet syndrome. Ultrastructural studies of bone marrow cells and megakaryocytes growing in culture from blood precursors. Am $\mathcal{F}$ Pathol 1981; 102: $10-9$.

22. Levy-Toledano S, Caen JP, Breton-Gorius J, et al. Gray platelet syndrome: $\alpha$-granule deficiency. Its influence on platelet function. $\mathcal{J} \mathrm{Lab}$ Clin Med 1981; 98: 831-48.

23. Nurden AT, Kunicki TJ, Dupuis D, Soria C, Caen JP. Specific protein and glycoprotein deficiencies on platelets isolated from two parients with the gray platelet syndrome. Blood 1982; 59: 709-18.

24. Leung LLK, Nachman RL. Complex formation of platelet thrombospondin with fibrinogen. 3 Clin Invest 1982; 70: 542-9.

25. Hiti-Harper J, Wohl H, Harper E. Platelet factor 4: an inhibitor of collagenase. Science 1978: 199: 991-2.

26. Kaplan DR, Chao FC, Stiles CD, Antoniades $H N$, Scher CD. Platelet $\alpha$-granules contain a growth factor for fibroblasts. Blood 1979; 53: 1043-52.

27. Glanzmann E. Hereditäre hämorrhagische Thrombasthenie. Ein Beitrag zur pathologie der blutplättchen. Jahrbuch der Kinderheilkunde $1918 ; 88: 1-42$.

28. Caen J, Cousin C. Le trouble d'adhésivité « in vitro des plaquettes dans la maladie de Willebrand et les thrombasthénies de Glanzmann. Essai d'interprétation. Nouv Rev Fr Hematol 1962, 2: 685-94.

29. Nurden AT, Caen JP. An abnormal platelet glycoprotein pattern in three cases of Glanzmann's thrombasthenia. Br 7 Haematol 1974; 28: 253-60.

30. Degos L, Dautigny A, Brouet JC, et al. A molecular defect in thrombasthenic platelets. 7 Clin Invest $1975 ; 56: 236-40$.

31. Hagen I, Nurden AT, Bjerrum OJ, Solum NO, Caen JP. Immunochemical evidence for protein abnormalitics in platelets from patients with Glanzmann's thrombasthenia and BernardSoulier syndrome. 7 Clin Invest 1980; 65: 722-31. 32. Pidard D, Montgomery RR, Bennett JS, Kunicki T J. Interaction of AP 2, a monoclonal antibody specific for the human platelet glycoprotein IIb/III a complex with intact platelets ; $7 \mathrm{Biol}$ Chem 1983; 258: 12582-6.

33. Lee $\mathrm{H}$, Nurden AT, Thomaïdis A, Caen J. Relationship between fibrinogen binding and the platelet glycoprotein deficiencies in Glanzmann's thrombasthenia type I and type II. Br 7 Haematol $1981 ; 48$ : 47-57.

34. Nachman RL, Leung LL. Complex formation of platelet membrane glycoprotein IIb and IIIa with fibrinogen. 7 Clin Invest 1982 ; 69: 263-9.

35. Nurden AT, Rosa JP, Legrand Y, Didry D, Pidard D, Parquet A. A variant of Glanzmann's thrombasthenia with abnormal glycoprotein $\mathrm{IIb} / \mathrm{III}$ a complexes in the platelet membrane. 3 Clin Invest 1985 (sous presse).

36. Caen JP. Variant thrombasthénie Paris-I Lariboisière. Trouble fonctionnel de l'agrégation des plaquettes humaines indépendant des glycoprotéines. $C R$ Acad Sci (Paris) $1985 ; 300$ : 417-20.

37. Georges JN, Nurden AT, Phillips DR. Molecular defects in interactions of platelets with the sévère. Par la suite, il fut montré dès 1962 que les plaquettes de ces sujets présentaient un défaut d'agrégation vis-à-vis de tous les agents inducteurs connus [28]; l'agglutination en présence de ristocétine était en revanche normale, parallèlement à une adhésion au subendothélium préservée. Comme dans le cas du syndrome de Bernard-Soulier, il fut bientôt possible de montrer que les plaquettes présentaient une anomalic membranaire : les glycoprotéines IIb et IIIa n'étaient pas détectables [29]. Grâce à un anticorps polyclonal IgG...L développé chez un sujet thrombasthénique polytransfusé, il fut possible d'analyser l'antigene absent au cours des thrombasthénies, par immunoprécipitation [30], ou immunoélectrophorèse en double dimension [31], et de montrer qu'il correspondait effectivement au complexe glycoprotéinique IIb/ IIIa. Par la suite, les études utilisant différents anticorps monoclonaux, dirigés en particulier contre ce complexe, ont permis de montrer qu'il était équimolaire, que les deux glycoprotéines étaient liées par un pont calcique et qu'il préexistait avant même l'état d'activation de la cellule [32]. Dès lors, la question consistait à savoir comment ces glycoprotéines intervenaient au cours de l'agrégation. Plusieurs expériences ont permis de répondre de plus en plus précisément. Tout d'abord, il fut possible de montrer que des plaquettes normales, mises en présence de l'anticorps IgG...L, présentaient un tableau de thrombasthénie : une relation fonctionnelle était dès lors mise au jour entre l'antigène glycoprotéinique IIb/ IIIa et la fonction d'agrégation.

Un peu plus tard, plusieurs équipes ont montré que ces plaquettes pathologiques étaient incapables de fixer le fibrinogène plasmatique [33], cofacteur nécessaire à l'agrégation [I7]. Le fait que le complexe glycoprotéinique constituait le site récepteur du fibrinogène plasmatique, était enfin prouvé directement in vitro, par l'étude de la fixation du fibrinogène, après isolement des glycoprotéines [34].

Le déficit plus ou moins notable en ce complexe permet de distinguer plusicurs types de thrombasthénic. Dans le type I, celui-ci n'est pas

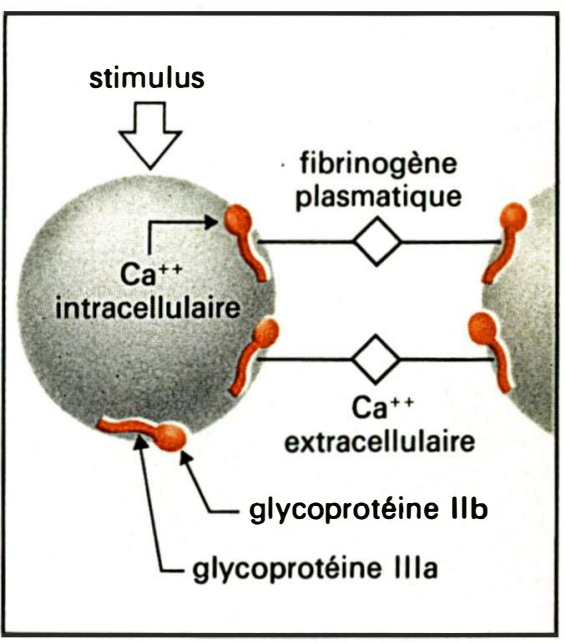

lïzure sa. Sihema de l'agregation plasurellative.

détectable, et l'on n'observe ni fixation de fibrinogène plasmatique, ni agrégation plaquettaire. Dans le type II, son taux est de $15 \%$ environ, entraînant une légère fixation $\mathrm{du}$ fibrinogène mais insuffisante pour provoquer une agrégation plaquettaire. Chez les sujets hétérozygotes, alors que le complexe est de l'ordre de $50 \%$ des valeurs normales, la fixation de fibrinogène et les agrégations plaquettaires sont déjà normales [33]. Ces faits illustrent l'importance de l'intensité du déficit de ce complexe dans l'apparition de l'anomalic fonctionnelle. Citons par ailleurs, l'importance immunogénique de ce complexe qui porte les antigènes spécifiques aux plaquettes : l'antigène $\mathrm{Pl}^{\mathrm{A}}{ }^{1}$ lié à la glycoprotéine IIIa, l'antigène Lek ${ }^{a}$ lié à la glycoprotéine IIb.

Le diagnostic prénatal et le dépistage des sujets hétérozygotes reposant sur ces tests immunologiques sont dès lors possibles : dans un avenir proche, l'isolement du gène codant pour ces glycoprotéines devrait permettre un diagnostic encore plus précoce...

Quoiqu'il en soit, c'est au niveau moléculaire que l'étude du rôle de ce complexe, au cours de la fonction d'agrégation, se poursuit actuellement. Récemment il a pu être identifié un type variant de thrombasthénic où l'anomalie fonction- 


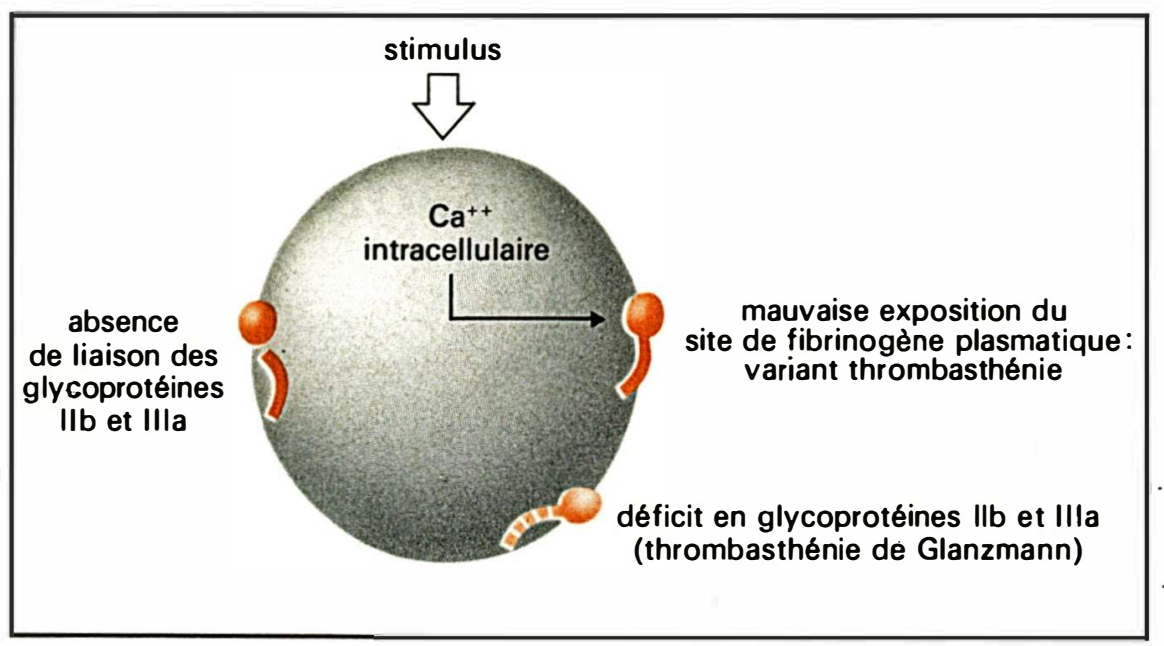

Figure 5 h. Thrombopalhies par défaut d'agrégation. nelle était due à l'absence de liaison de ces deux glycoprotéines IIb et IIIa pour former un complexe grâce à un pont calcique [35]; de même au cours du variant thrombasthénie Paris-I - Lariboisic̀re [36], c'est le défaut d'exposition du site récepteur du fibrinogène au sein du complexe glycoprotéinique, après l'étape d'activation, qui est responsable du tableau hémorragique. La description de tels variants vient donc confirmer précisément les données des travaux fondamentaux. L'étude toujours plus détaillée de ces tableaux de thrombasthénie devrait permettre dans les prochaines années la description précise des modifications membranaires qui surviennent au cours de l'activation plaquettaire afin de permettre l'agrégation. Une meilleure compréhension de ces phénomènes pourrait provoquer l'avènement d'une nouvelle classe de composés à action antiagrégante, donc antithrombotique (figures $5 a$ et $5 b$ ).

\section{Autres thrombopathies}

Les plaquettes jouent un rôle catalytique important de l'hémostase plasmatique, offrant une surface cellulaire riche en phospholipides qui permet l'activation du facteur $\mathrm{X}$ ou de la prothrombine. Dans la littérature, trois cas de thrombopathies ont été rapportés, où cette fonction pouvait être perturbée [37]; citons en particulier le cas où la démonstration fut donnée du fait que les plaquettes étaient dépourvues des sites récepteurs pour le facteur Va. Au cours de ces thrombopathies, les manifestations hémorragiques ressemblent à celles ducs à un déficit de la coagulation mais peuvent être prévenues par l'administration de plaquettes.

Ainsi, bien qu'exceptionnelles, ces thrombopathies permettent-elles de confirmer le rôle essentiel joué par les plaquettes au cours de la coagulation plasmatique.

\section{Conclusion}

Par la description des thrombopathies constitutionnelles, il nous a été possible de montrer les progrès et les limites de la connaissance actuelle du rôle de ces cellules originales sans noyau que sont les plaquettes. Ce rôle nous semble aller bien au-delà du champ de l'hémostase pour s'étendre aux domaines de l'inflammation, de la cancérologie ou de la neurophysiologie...

\section{Summary}

Constitutional thrombocytopathies were described concerning each step of primary haemostasis. Adhesion to subendothelium, cellular activation, granular secretion or platelet aggregation may be defective. The exact mechanisms responsible for these abnormalities were extensively studied and are now often known at the molecular level. These studies permitted a better understanding of primary haemostasis leading to progress in antithrombotic therapy.

\section{TIRES A PART}

J.P. Caen : département d'angio-hématologie, hôpital Lariboisière, 6 , rue Guy-Patin, 750 oro Paris. 